\title{
Design and Realization of Micronet Page Based on H5 Media Platform
}

\author{
Wei Gao ${ }^{\text {a,* }}$, Zhen Chen \\ Sichuan University of Media and Communications, Chengdu, 611745, China \\ a gw510230@163.com \\ *corresponding author
}

Keywords: H5, Melt Media, Micronet Page

\begin{abstract}
In the environment where the information is almost and the new media industry is developing continuously, the microweb page has become one of the important media types of the mobile side. Among them, H5 digital content products are common in the design and manufacture of micronet pages, and also have strong practicability. This paper firstly expounds the principles and key points of the design of the micro-net page based on the $\mathrm{H} 5$ media platform, and then focuses on the research object of the propaganda web page produced by the H5 micro-page contest organized by a new media major in Hunan University.
\end{abstract}

\section{Introduction}

With the continuous development of contemporary social economy, the arrival of the new media era based on mobile communication network technology has changed people's life and consciousness. The micronet page based on HTML5's melt media platform is gradually becoming one of the mainstream media presentation forms on the mobile side, which provides a new choice for the operation and promotion of new media practitioners. The design and production of H5 micropages not only need to rely on good aesthetic awareness to do a good job of visual design, but also from the marketing, promotion, customer service and other aspects of functional design, and finally through the H5 micropages to achieve the relevant marketing services.

\section{Principles and Key Points for the Design of Micro Web Pages Based on the H5 Fusion Media Platform}

\subsection{Aesthetic}

Meet people's aesthetic pursuit, accord with the aesthetic habits of the audience, is the first key point of micro-web page design. Nowadays, most $\mathrm{H} 5$ media platforms on the market have a variety of templates for users to choose from, which have a bright design for color matching, artistic sense and dynamic effects[1]. At the same time, related platforms support pictures, fonts, dynamic effects of random collocation, so that users have a broader creative space. With the support of such a powerful $\mathrm{H} 5$ melt media platform, the author needs to have a good aesthetic ability, on the one hand to understand what kind of visual design can make people feel amazing, on the other hand, to understand the aesthetic habits of the relevant audience of the micro web pages made by themselves.

\subsection{Functionality}

In the new media environment, the design requirements of micro web pages are aimed at certain application requirements. For example, the brand promotion of enterprises, new products marketing, online activities and so on, can use micro-pages to promote, or even complete some information collection, online business purposes. The design of micro-web page based on H5 fusion media platform needs to make clear the purpose of the web page, and then add some functions to it. Nowadays, the design software of some $\mathrm{H} 5$ fusion media platform has corresponding functional modules, such as phone number login, user information filling and collection, jump out link and so on. From the design and implementation of the whole web page, 
whether it is a copy, picture or information presentation process, it needs to be based on the realization of the purpose of web application, such a micro-web page is practical.

\subsection{Conciseness}

Whether as a marketing promotion or user-run purpose of micro-web page, need to meet the requirements of simplicity, easy to understand, easy to operate. From the design point of view, the number of words is not easy to copy, each page, a version of the copy should be concise to ensure that the first time to obtain accurate information, and colour collocation, graphics design should not be too complex, so as not to affect the user's reading. From a functional point of view, the microweb platform information presentation, dynamic effects, audio playback should remain smooth, no Carton. At the same time, the user's click, operation is convenient enough, the page response is fast enough. This requires careful screening of the $\mathrm{H} 5$ media fusion platform, selection of more mature technology platform, on the basis of the user interaction design, comprehensively enhance the user's viewing, operation experience[2].

\section{Selection and Evaluation of the H5 Media Platform}

The $\mathrm{H} 5$ media fusion platform has the powerful design and editing function of micronet pages, and it can also realize the direct docking with different sizes, operating system and new media communication channels, which is very practical. At present, the mainstream H5 media design software has the following:

\subsection{Yi Jixiu}

The software is a H5 microweb design and production tool mainly aimed at the marketing of mobile Internet, which fully absorbs a variety of marketing schemes with mature PC, and makes a reasonable simplification according to the actual situation and application requirements of the mobile phone, which allows users to make multiple types of $\mathrm{H} 5$ microweb pages directly on the PC and even the mobile phone. Yi's advantage lies in having a large number of mature templates, which are diverse in style and suitable for different marketing needs, which can enable users to make various microweb pages of DIY in a short period of time without mastering complex programming techniques.[3]. At the same time, the micro-web pages made by Yi enterprise show can be shared and spread directly on the current mainstream new media social platform, and the convenience is extremely high. However, relatively speaking, Yi enterprise show's personalized creation space is small, the function is limited, is not suitable for making the micro web page with high complexity and high interactive setting requirements.

\subsection{Epub360}
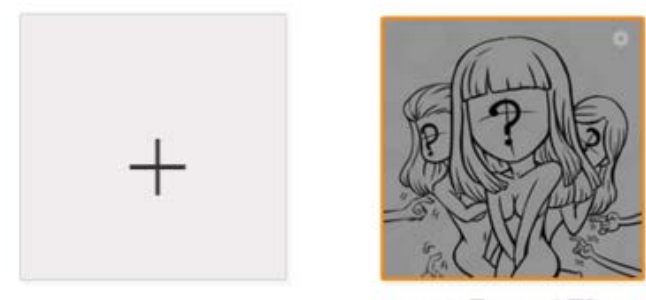

copy_180震Young席国队....
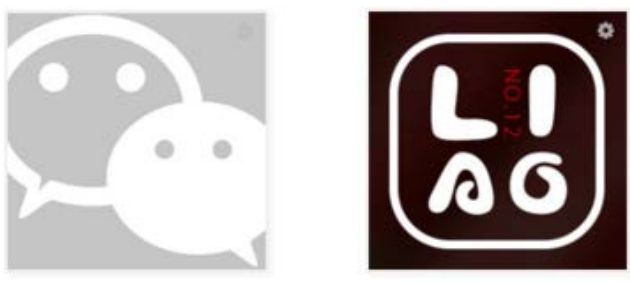

Figure 1 Epub360 design interface 
Epub360 is a professional H5 media fusion tool for new media interaction designers and Internet professionals, which not only enables interface, animation and interaction design, but also has powerful logic functions[4]. as shown in figure 1, the epub360 has powerful logical programming and visual design capabilities to make and publish more original class micropages. The platform can realize animation control, interaction setting and social application simultaneously, and can also cooperate with users to collect data and analyze applications.

\section{The Design and Realization Process of Micronet Page Based on H5 Fusion Media Platform}

A university in Hunan opened a new media major in 2017, and in March 2019, students of the school's new media major jointly organized the $\mathrm{H} 5$ microweb design and production competition with its computer software technology institute. To promote the event and engage students and teachers, the event's organization and publicity team designed and produced a web page using the epub360 media platform.

\subsection{Determining the Subject of the Web Page}

First of all, the event group met to discuss the theme of the micro-page production, namely, to promote the $\mathrm{H} 5$ micro-page design and production competition to attract the participation of teachers and students, and to provide participants with a reference template, which has the effect of throwing a brick and a brick. Specifically, the micro-page is divided into three sections, the theme of publicity and process, professional introduction, message wall. First of all, the activity theme publicity and process interface needs to show the activity slogan, the main copy, the activity content and the time and so on information; secondly, the college introduction interface needs to include the specialized brief introduction, the specialized course, the employment direction and so on; finally, the message wall interface collects the student teacher message. In addition, the entire micro-web page added background music module, music selected the school song.

\subsection{Establishment of Micro-Web Style and Concept}

The face of the micro-page is the whole school teachers and students, so the overall visual design needs to conform to the aesthetic of contemporary college students, in tone is not too much, need to have a certain sense of art. And the micro-page production team also found the school cartoon students to help design and produce related cartoon characters and pictures, as the main elements of the micro-net page. At the same time, functional design and dynamic design should not be too complex, so that different performance of mobile devices can run smoothly. Finally, in the writing and design of copywriting, we need to be concise and focused[5].

\subsection{Features of Micro-Page Works}

Through the epub360 media platform, the school's new media students and computer software students to cooperate, selected the youth bright yellow as the main tone. First of all, the theme page of the micronet page see figure 2," start to sign up "theme words to use the dynamic effect, simple and clear transmission of the main information; cartoon theme style and contemporary college students aesthetic; the ">" icon at the bottom of the page also used the dynamic effect of exploration, guide users to draw the page down.

Secondly, the introduction of the micropage activity flow according to the process using the dynamic effect displayed in turn, so that users can simply understand the way to participate in the activity, but also add some microweb page design schematic, so that students and teachers can see the charm of the micropage. Then, the professional introduction interface of the web page continues the same visual style (see figure 3), introducing the new media specialty with the key words close to the language habits of college students, simple, clear and attractive. The purpose of this section is to make people in and out of the school better understand the new media specialty, and the related characters are made to be clickable. After clicking, users can further read the details of the new media specialty in the school, and understand the course, professional employment direction and other extended reading content. Finally, the message wall adopts the message wall module of the 
epub360 melt media platform, and the "sharing circle of friends" button is added in the prominent position in the middle, so that the user can share the activity page to the circle of friends one by one to expand the publicity.

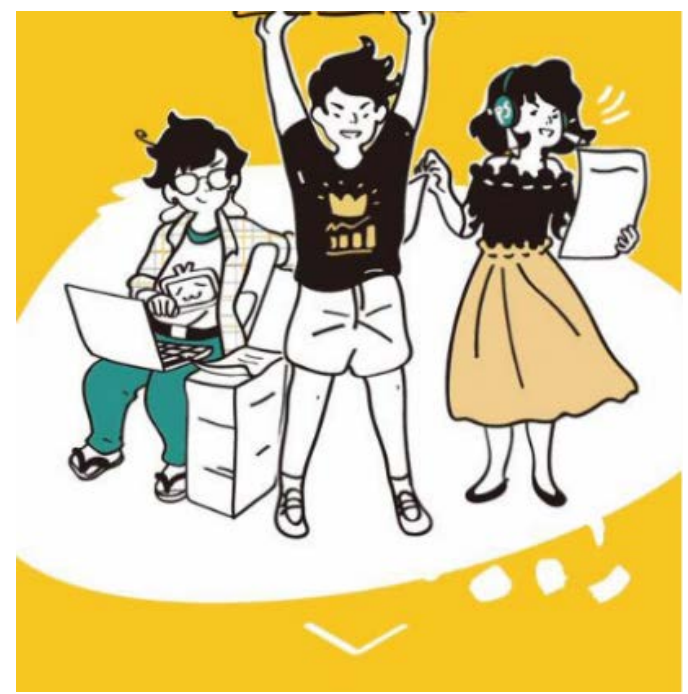

Figure 2 Micropage theme page

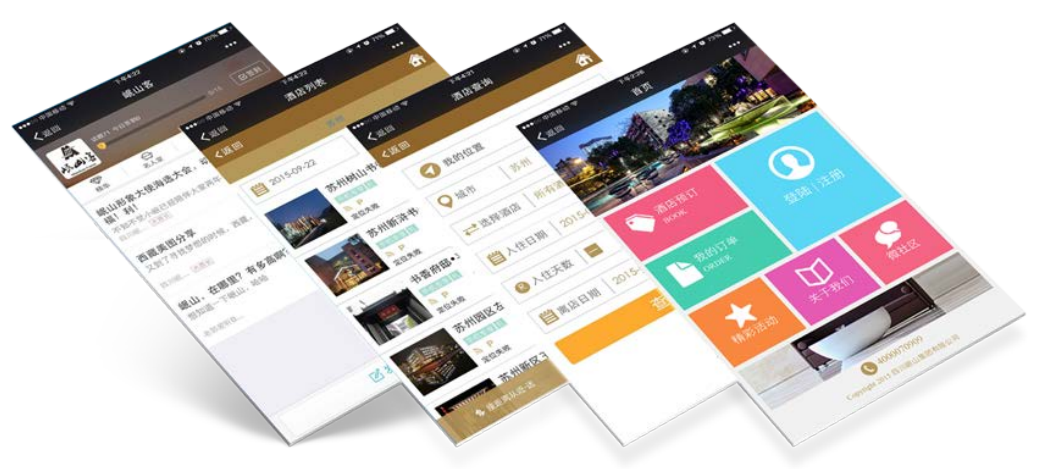

Figure 3 Micropage professional presentation page

In addition, in order to better achieve the relevant functions of microweb pages, the design team also previewed the completed microweb pages on the epub360 platform, and then shared to wechat, qq and other new media social platforms to apply, to determine the overall operation of stable and smooth, interactive features without loopholes, and then go online to promote.

\section{Conclusion}

To sum up, in the new media age, mobile Internet marketing and user services have more diversified technical support, based on H5 The use value of the micronet page produced by the melt media platform is extremely high. In the design and production of micropages, it is necessary to select the appropriate $\mathrm{H} 5$ media production platform or software on the basis of clear theme content and main audience characteristics, and then adhere to the principle of beauty, simplicity and practicality, and do a good job of visual design, functional design and interactive design of micropages. Only in this way, can we complete the more attractive microweb pages to meet the needs of marketing promotion, customer service and so on.

\section{References}

[1] Zhou, Ji. Mobile terminal h5 ad micro web design and implementation — take university student advertising competition as an example. Tiangong, no. 6, pp. 25, 2019.

[2] Shanghai, Diyang., Industrial Co., Ltd. A WeChat H5 web page making method and system: 
CN201710498020.X, no. 10-03, 2017.

[3] Wang, Peizhi. Scene Video's H5 Innovation —— Take "Wang Xiaoyi's Circle of Friends" as an example. China Newspapers , no. 7, pp. 46-47, 2017.

[4] Zheng, Shuyi. The Application of Empathy Design in H5 Advertising — A Case Study of H5 Advertising Design in Caran Zen Life Hall. Zhejiang: Zhejiang University of Technology, 2018.

[5] Yu, Weiya., Jiao, Xufeng. How to Make Use of the New Media Platform to Report on Poverty Alleviation — Take Xinhua News Agency's Interactive H5" To Fight for the Fight against Poverty "as an example. Media, no. 21, pp. 59-60, 2019. 